\title{
1-Quasi Total Fuzzy Graph and Its Total Coloring
}

Fekadu Tesgera Agama*, Venkata Naga Srinivasa Rao Repalle

Department of Mathematics, College of Natural and Computational Science, Wollega University, Nekemte, Ethiopia

Email address:

fekadutsgr.2019@gmail.com (F. T. Agama),rvnrepalle@gmail.com(V. N. S. R. Repalle)

${ }^{*}$ Corresponding author

\section{To cite this article:}

Fekadu Tesgera Agama, Venkata Naga Srinivasa Rao Repalle. 1-Quasi Total Fuzzy Graph and its Total Coloring. Pure and Applied Mathematics Journal. Vol. 9, No. 1, 2020, pp. 9-15. doi: 10.11648/j.pamj.20200901.12

Received: November 27, 2019; Accepted: December 21, 2019; Published: January 17, 2020

\begin{abstract}
The fuzzy graph theory, its properties, total coloring and applications are currently climbing up. With this concept of fuzzy graph, total fuzzy graph is defined and its properties as well as fuzzy total colorings have been well discussed and studied. Similarly the theory of crisp graph, its properties, applications and colorings are well considered. Moreover, 1-quasi total graphs for crisp graphs, their properties and colorings were discussed by some researchers and the bounds for its total coloring have been established. In this manuscript, from the concept of fuzzy graph we introduced the definition of 1-quasi total graph for fuzzy graphs. To elaborate the definition we provide practical example of fuzzy graph and from this graph we construct the 1-quasi total fuzzy graph of the given fuzzy graph, so that the definition to be meaning full and their relationships can be easily observed from the sketched graphs. In addition some theorems related to the properties of 1-quasi total fuzzy graphs are stated and proved. The results of these theorems are compared with the results obtained from total fuzzy graphs, so that the differences and similarities that 1-quasi total fuzzy graph can have with that of total fuzzy graphs are revealed. Moreover, we define 1-quasi total coloring of fuzzy total graphs and give an example of total coloring of 1-quasi total graphs.
\end{abstract}

Keywords: Fuzzy Graph, Total Fuzzy Graph, 1-Quasi Total Fuzzy Graph, Total Coloring

\section{Introduction}

After its emerging, the graph theory rapidly moved in to the main stream of mathematics as it has application in diverse fields of science and computer [1-2]. The total coloring of graphs were introduced by Behazad (1965) after Harry (1972) made chops on the concept of total graphs [3-4] D. Muthuramakrishnan and G. Jayaraman (2018) studied the total chromatic number of total graphs [5]. The definition of quasi-total graph is given on the paper by D. V. S. Sastry (1984) and R. V. N. SirnivasaRao, etal has introduced 1-quasi total graphs and bounds for its total chromatic number [6-7]. After Zadeh's paper on fuzzy sets, Rosenfeld (1975) introduced fuzzy graphs [8-9].

Later on, Bhattacharya [10] gave some remarks on fuzzy graphs, and some operations on fuzzy graphs were introduced by Mordeson J. N. and Peng C. S. [11]. As an advancement fuzzy coloring of fuzzy graph was defined by Eslahchi and Onagh in 2004, and later developed by them as Fuzzy vertex coloring in 2006 [12]. Lavanay. S and Sattanathan extended the concept of fuzzy vertex coloring in to a family of fuzzy sets [13]. S. Kavitha and S. Lavanya defined the total fuzzy graph and studied total chromatic number of total graphs of fuzzy graphs [14].

Total coloring of 1-quasi total graph for crisp graph was studied and total fuzzy graphs and its total chromatic number has been already established. This article addresses the following:

i. Define 1-quasi total fuzzy graph and elaborate it by examples.

ii. Discuss and proof some properties of 1-quasitotal fuzzy graphs and compare the result with the properties of total fuzzy graphs.

iii.Determine the total chromatic number of 1-quasi total fuzzy graph and justify it by example.

\section{Preliminaries}

Under this topic we give the definition of some concepts which help us as a prerequisite to present the main body of the manuscript. Most of the concepts are from [1-18].

Definition 2.1: A fuzzy graph is defined as an ordered triple $G=(V, \sigma, \mu)$, where $V$ is the set of vertices 
$\left\{v_{1}, v_{2}, \ldots, v_{n}\right\}, \sigma$ is a fuzzy subset of $V$, such that $\sigma: V \rightarrow$ $[0,1]$ and $\mu$ be a fuzzy relation on $\sigma$ with $\mu: V \rightarrow[0,1]$ and that $\mu: V \times V \rightarrow[0,1] \quad$ such that $\mu(u, v) \leq \sigma(u) \Lambda \sigma(v) \forall u, v \in V$.

Definition 2.2: The underlying crisp graph of the fuzzy graph $G=(V, \sigma, \mu)$ is denoted by $G^{*}=(V, E)$, where $E \subseteq V \times V$. The crisp graph $(V, E)$ is a special fuzzy graph $G$ with each vertex and each edges of $G$ has the same degree of membership equal to 1 .

Definition 2.3: Let $G=(V, \sigma, \mu)$ be a fuzzy graph with the underlying set $V$. Then, the order of $G$ denoted by $\operatorname{Order}(G)$ is defined as:

$$
\operatorname{Order}(G)=\sum_{u \in V} \sigma(u)
$$

and size of $G$ denoted by $S$ i z $e(G)$ and defined as:

$$
\operatorname{Size}(u)=\sum_{u, v \in V} \mu(u, v)
$$

Definition 2.4: Let $G=(V, \sigma, \mu)$ be a fuzzy graph. The degree of a vertex $u \in V$ is defined as

$$
d_{G}(u)=\sum_{v \neq u, v \in V} \mu(u, v)
$$

Definition 2.5: Let $G=(V, \sigma, \mu)$ be a fuzzy graph. The busy value of the vertex $v$ in $G$ is $D(v)=\sum_{i} \sigma(v) \Lambda \sigma\left(v_{i}\right)$ where $v_{i}$ are neighbors of $v$ and the busy value of $G$ is

$$
D(G)=\sum_{i} D\left(v_{i}\right)
$$

where $v_{i}$ are the vertexes of $G$.

Definition 2.6: If $\mu(u, v)>0$, then $u$ and $v$ are said to be adjacent to each other and lie on the edge, $e=(u, v)$. A path $\rho$ in a fuzzy graph $G=(V, \sigma, \mu)$ is a sequence of distinct nodes $v_{0}, v_{1}, v_{2}, \ldots, v_{n}$ such that $\mu\left(v_{i-1}, v_{i}\right)>0,1 \leq i \leq n$. Here $n$ is called the length of the path.

Definition 2.7: If $u, v$ are vertices in $G$ and if they are connected by means of a path, then the strength of that path is defined as $\Lambda_{i=1}^{n} \mu\left(v_{i-1}, v_{i}\right)$. If $u, v$ are connected by means of paths of length $k$, then

$$
\begin{gathered}
\mu^{k}(u, v)=\sup \left\{\mu\left(u, v_{1}\right) \Lambda \mu\left(v_{1}, v_{2}\right) \Lambda \mu\left(v_{2}, v_{3}\right)\right. \\
\left.\Lambda \ldots \Lambda \mu\left(v_{k-1}, v\right): u, v_{1}, v_{2}, \ldots, v_{k-1}, v \in V\right\}
\end{gathered}
$$

If $u, v \in V$, then the strength of connectedness between $u$ and $v$ is,

$$
\mu^{\infty}(u, v)=\sup \left\{\mu^{k}(u, v): k=1,2, \ldots\right\}
$$

Definition 2.8: Let $G=(V, \sigma, \mu)$ be a fuzzy graph. Then, $G$ is said to be connected if $\mu^{\infty}(u, v)>0$ for all $u, v \in \sigma^{*}$. An $\operatorname{arc}(u, v)$ is said to be a strong arc if $\mu(u, v) \geq \mu^{\infty}(u, v)$ and a node $u$, is said to be an isolated node, if $\mu(u, v)=$ 0 for all $u \neq v$.

Definition 2.9: $G=(V, \sigma, \mu)$ is a fuzzy cycle if and only if $\left(\sigma^{*}, \mu^{*}\right)$ is cycle and there does not exist a unique $(x, y) \in \mu^{*}$ such that $\mu(x, y)=\Lambda\left\{\mu(\mathrm{u}, \mathrm{v}):(\mathrm{u}, \mathrm{v}) \in \mu^{*}\right\}$.

Definition 2.10: A family $\Gamma=\left\{\gamma_{1}, \gamma_{2}, \gamma_{3}, \ldots, \gamma_{k}\right\}$ of fuzzy sets on $V \cup E$ is called a $k$-fuzzy total coloring of $G=$ $(V, \sigma, \mu)$, if:

a) $\operatorname{Max}\left\{\gamma_{i}(v)\right\}=\sigma(v)$ for all $v \in V$ and

b) $\operatorname{Max}\left\{\gamma_{i}(u, v)\right\}=\mu(u, v)$ for all edges $(u, v) \in E$

c) $\gamma_{i} \Lambda \gamma_{j}=0$

d) For every adjacent vertices $u, v$ of $G$, $\operatorname{Min}\left\{\gamma_{i}(u), \gamma_{i}(v)\right\}=0$.

The least value of $k$ for which there exists a $k$-fuzzycoloring is called the fuzzy total chromatic number of $G$ and is denoted by $\chi_{T}^{f}(G)$.

\section{1-Quasi Fuzzy Total Graph}

In this section we introduce the definition of 1-quasi fuzzy total graph and draw the 1-quasi fuzzy total graph of a given fuzzy graph.

Definition 3.1: Let $G=(V, \sigma, \mu)$ be a fuzzy graph with its underlying set $V$ and crisp graph $G^{*}=\left(\sigma^{*}, \mu^{*}\right)$. The pair $Q_{1} T_{f}(G)=\left(\sigma_{Q_{1} T_{f}}, \mu_{Q_{1} T_{f}}\right)$ of the fuzzy graph $G$ is defined as follows:

Let the node set of $Q_{1} T_{f}(G)$ be $V \cup E$, where $V$ is the vertex set and $E$ is the edge set of the underlying crisp graph. The fuzzy subset $\sigma_{Q_{1} T_{f}}$ is defined on $V \cup E$ as:

$$
\begin{gathered}
\sigma_{Q_{1} T_{f}}(u)=\sigma(u), \text { if } u \in V \\
\sigma_{Q_{1} T_{f}}(e)=\mu(e), \text { if } e \in E
\end{gathered}
$$

The fuzzy relation $\mu_{Q_{1} T_{f}}$ is defined on $(V \cup E) \times(V \cup E)$, called edges of $Q_{1} T_{f}(G)$ as:

$$
\mu_{Q_{1} T_{f}}(u, v)=\mu(u, v), \text { if } u, v \in V
$$

$\mu_{Q_{1} T_{f}}\left(e_{i}, e_{j}\right)=\mu\left(e_{i}\right) \Lambda \mu\left(e_{j}\right)$, if $e_{i}$ and $e_{j}$ have a node in com mon between them $=0$, Otherwise

By definition; $\mu_{Q_{1} T_{f}}(u, v) \leq \sigma_{Q_{1} T_{f}}(u) \Lambda \sigma_{Q_{1} T_{f}}(v)$ for all $u, v \in V \cup E$. Hence, $\mu_{Q_{1} T_{f}}$ is a fuzzy relation on the fuzzy subset $\sigma_{Q_{1} T_{f}}$. Thus, the pair $Q_{1} T_{f}\left(\sigma_{Q_{1} T_{f}}, \mu_{Q_{1} T_{f}}\right)$ is a fuzzy graph, and it is termed as 1-Quasi total fuzzy graph of $G$.

Example 3.1: Consider the fuzzy graph $G=(V, \sigma, \mu)$ where its underlying crisp graph $G^{*}=(V, E)$ has vertex set $V=\left\{v_{1}, v_{2}, v_{3}\right\}$ and edge set $E=\left\{v_{1} v_{2}, v_{2} v_{3}, v_{3} v_{1}\right\}$. Let the fuzzy vertex set defined on $V$ be as follows:

$\sigma\left(v_{1}\right)=0.4, \sigma\left(v_{2}\right)=0.5, \sigma\left(v_{3}\right)=0.7$.

Let the fuzzy relation defined on the fuzzy edge set be follows:

$$
\mu\left(v_{1}, v_{2}\right)=0.2, \mu\left(v_{2}, v_{3}\right)=0.4, \mu\left(v_{3}, v_{1}\right)=0.4
$$

Since, $\mu\left(v_{i}, v_{j}\right) \leq \sigma\left(v_{i}\right) \Lambda \sigma\left(v_{j}\right)$ for all $v_{i}, v_{j} \in V$, the graph $G=(V, \sigma, \mu)$ is a fuzzy graph and its graph is as shown in the figure 1. 


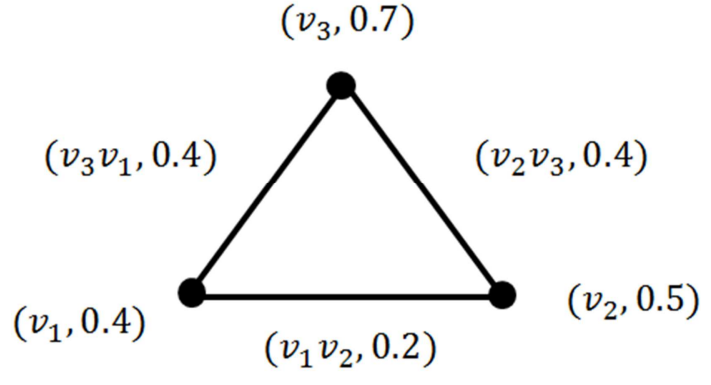

Figure 1. Fuzzy Graph.

Now, let us construct the 1-quasi total fuzzy graph of the fuzzy graph in the example as follows:

That is, $Q_{1} T_{f}\left(\sigma_{Q_{1} T_{f}}, \mu_{Q_{1} T_{f}}\right)$ of the fuzzy graph $G$, where the node set of $Q_{1} T_{f}$ is $V \cup E$, which is the set $\left\{v_{1}, v_{2}, v_{3}, v_{1} v_{2}, v_{2} v_{3}, v_{3} v_{1}\right\}$. We define the fuzzy subset $\delta_{Q_{1} T_{f}}$ as follows:

$$
\begin{aligned}
& \sigma_{Q_{1} T_{f}}(u)=\sigma(u), \text { if } u \in V \\
& \sigma_{Q_{1} T_{f}}(e)=\mu(e), \text { if } e \in E .
\end{aligned}
$$

Hence, we have the following fuzzy subsets $\sigma_{Q_{1} T}$ :

$$
\begin{gathered}
\sigma_{Q_{1} T_{f}}\left(v_{1}\right)=\sigma\left(v_{1}\right)=0.4, \sigma_{Q_{1} T_{f}}\left(v_{2}\right)=\sigma\left(v_{2}\right)=0.5 \\
\sigma_{Q_{1} T_{f}}\left(v_{3}\right)=\sigma\left(v_{3}\right)=0.7 \\
\sigma_{Q_{1} T_{f}}\left(v_{1} v_{2}\right)=\mu\left(v_{1}, v_{2}\right)=0.2, \sigma_{Q_{1} T_{f}}\left(v_{2} v_{3}\right) \\
=\mu\left(v_{2}, v_{3}\right)=0.4, \sigma_{Q_{1} T_{f}}\left(v_{3} v_{1}\right)=\mu\left(v_{3}, v_{1}\right)=0.4
\end{gathered}
$$

The fuzzy relations $\mu_{Q_{1} T_{f}}$ will be as follows:

$$
\mu_{Q_{1} T_{f}}(u, v)=\mu(u, v) \text {, if } u, v \in V
$$

$\mu_{Q_{1} T_{f}}\left(e_{i}, e_{j}\right)=\mu\left(e_{i}\right) \Lambda \mu\left(e_{j}\right)$, if $e_{i}$ and $e_{j}$ have a node in common between them $=0$, otherwise

Hence,

$$
\begin{gathered}
\mu_{Q_{1} T_{f}}\left(v_{1}, v_{2}\right)=\mu\left(v_{1}, v_{2}\right)=0.2, \\
\mu_{Q_{1} T_{f}}\left(v_{2}, v_{3}\right)=\mu\left(v_{2}, v_{3}\right)=0.4 \\
\mu_{Q_{1} T_{f}}\left(v_{3}, v_{1}\right)=\mu\left(v_{3}, v_{1}\right)=0.4 \\
\mu_{Q_{1} T_{f}}\left(v_{1} v_{2}, v_{2} v_{3}\right)=\mu\left(v_{1} v_{2}\right) \Lambda \mu\left(v_{2} v_{3}\right)=0.2 \\
\mu_{Q_{1} T_{f}}\left(v_{1} v_{2}, v_{3} v_{1}\right)=\mu\left(v_{1} v_{2}\right) \Lambda \mu\left(v_{3} v_{1}\right)=0.2 \\
\mu_{Q_{1} T_{f}}\left(v_{2} v_{3}, v_{3} v_{1}\right)=\mu\left(v_{2} v_{3}\right) \Lambda \mu\left(v_{3} v_{1}\right)=0.4
\end{gathered}
$$

Since, $\quad \mu_{Q_{1} T_{f}}\left(v_{i}, v_{j}\right) \leq \sigma_{Q_{1} T_{f}}\left(v_{i}\right) \Lambda \sigma_{Q_{1} T_{f}}\left(v_{j}\right)$ for all $v_{i}, v_{j} \in V \cup E$, the graph $Q_{1} T_{f}\left(\sigma_{Q_{1} T_{f}}, \mu_{Q_{1} T_{f}}\right)$ is a fuzzy graph and from the above node sets $V \cup E$, fuzzy subsets $\sigma_{Q_{1} T_{f}}$ and fuzzy relations $\mu_{Q_{1} T_{f}}$, the graph of 1-quasi total fuzzy graph of $G$ is as shown in figure 2 .

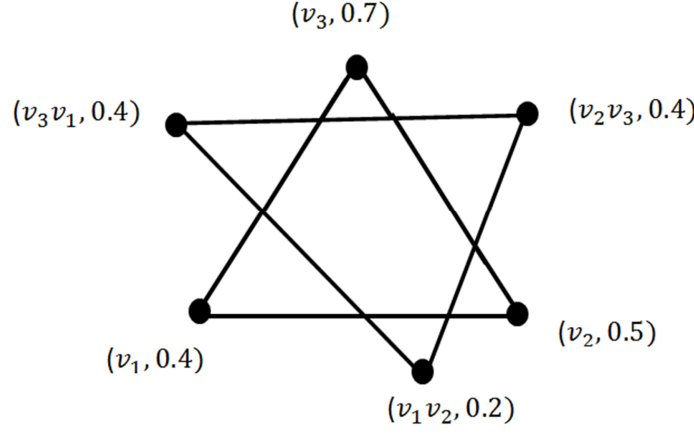

Figure 2. 1-Quasi fuzzy total graph.

Example 3.2: Consider the fuzzy graph $G=(V, \sigma, \mu)$ with the fuzzy vertex set;

$$
\sigma\left(v_{1}\right)=0.3, \sigma\left(v_{2}\right)=0.4, \sigma\left(v_{3}\right)=0.6, \sigma\left(v_{4}\right)=0.8
$$

and fuzzy edge set:

$$
\begin{gathered}
\mu\left(v_{1}, v_{2}\right)=0.2, \mu\left(v_{2}, v_{3}\right)=0.3 \\
\mu\left(v_{3}, v_{4}\right)=0.5, \mu\left(v_{4}, v_{1}\right)=0.1
\end{gathered}
$$

Since, $\mu\left(v_{i}, v_{j}\right) \leq \sigma\left(v_{i}\right) \Lambda \sigma\left(v_{j}\right)$ for all $v_{i}, v_{j} \in V$, the graph $G=(V, \sigma, \mu)$ is a fuzzy graph and its graph is as shown in the figure 3 below.

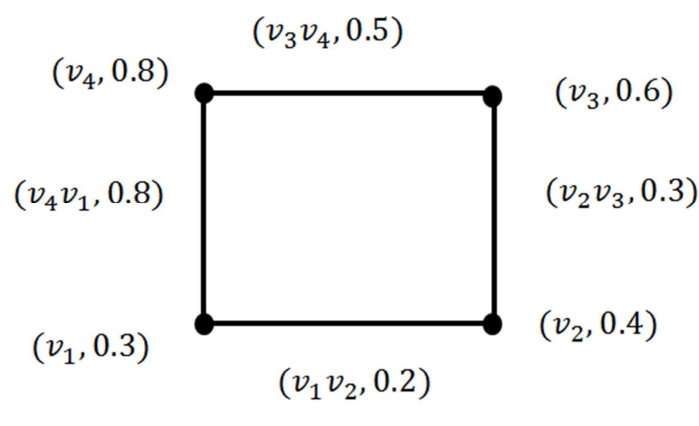

Figure 3. Fuzzy Graph.

Now, from the fuzzy graph in example 3 the 1-quasi total fuzzy graph of $G, Q_{1} T_{f}\left(\sigma_{Q_{1} T_{f}}, \mu_{Q_{1} T_{f}}\right)$ will be defined as follows:

i. Fuzzy vertex set $\sigma_{Q_{1} T_{f}}$ is as follows:

$$
\begin{aligned}
& \sigma_{Q_{1} T_{f}}(u)=\delta(u), \text { if } u \in V \\
& \sigma_{Q_{1} T_{f}}(e)=\mu(e), \text { if } e \in E .
\end{aligned}
$$

Hence;

$$
\begin{gathered}
\sigma_{Q_{1} T_{f}}\left(v_{1}\right)=\sigma\left(v_{1}\right)=0.3, \\
\sigma_{Q_{1} T_{f}}\left(v_{2}\right)=\sigma\left(v_{2}\right)=0.4, \\
\sigma_{Q_{1} T_{f}}\left(v_{3}\right)=\sigma\left(v_{3}\right)=0.6, \\
\sigma_{Q_{1} T_{f}}\left(v_{4}\right)=\sigma\left(v_{4}\right)=0.8 \\
\sigma_{Q_{1} T_{f}}\left(v_{1} v_{2}\right)=\mu\left(v_{1}, v_{2}\right)=0.2,
\end{gathered}
$$




$$
\begin{gathered}
\sigma_{Q_{1} T_{f}}\left(v_{2} v_{3}\right)=\mu\left(v_{2}, v_{3}\right)=0.3, \\
\sigma_{Q_{1} T_{f}}\left(v_{3} v_{4}\right)=\mu\left(v_{3}, v_{4}\right)=0.5, \\
\sigma_{Q_{1} T_{f}}\left(v_{4} v_{1}\right)=\mu\left(v_{4}, v_{1}\right)=0.1
\end{gathered}
$$

ii. The fuzzy edge set $\mu_{\mathrm{Q}_{1} \mathrm{~T}_{\mathrm{f}}}$ is as follows:

$$
\mu_{Q_{1} T_{f}}(u, v)=\mu(u, v) \text {, if } u, v \in V
$$

$\mu_{Q_{1} T_{f}}\left(e_{i}, e_{j}\right)=\mu\left(e_{i}\right) \Lambda \mu\left(e_{j}\right)$, if $e_{i}$ and $e_{j}$ have a node in common between them $=0$, Otherwise

Hence,

$$
\begin{gathered}
\mu_{Q_{1} T_{f}}\left(v_{1}, v_{2}\right)=\mu\left(v_{1}, v_{2}\right)=0.2, \mu_{Q_{1} T_{f}}\left(v_{2}, v_{3}\right)= \\
\mu\left(v_{2}, v_{3}\right)=0.3 \\
\mu_{Q_{1} T_{f}}\left(v_{3}, v_{4}\right)=\mu\left(v_{3}, v_{4}\right)=0 . \\
\mu_{Q_{1} T_{f}}\left(v_{4}, v_{1}\right)=\mu\left(v_{4}, v_{1}\right)=0.1
\end{gathered}
$$$$
\mu_{Q_{1} T_{f}}\left(v_{1} v_{2}, v_{2} v_{3}\right)=\mu\left(v_{1} v_{2}\right) \Lambda \mu\left(v_{2} v_{3}\right)=0.2 \Lambda 0.3=0.2
$$$$
\mu_{Q_{1} T_{f}}\left(v_{1} v_{2}, v_{3} v_{4,}\right)=\mu\left(v_{1} v_{2}\right) \Lambda \mu\left(v_{3} v_{4}\right)=0
$$$$
\mu_{Q_{1} T_{f}}\left(v_{1} v_{2}, v_{4} v_{1}\right)=\mu\left(v_{1} v_{2}\right) \Lambda \mu\left(v_{4} v_{1}\right)=0.2 \Lambda 0.1=0.1
$$$$
\mu_{Q_{1} T_{f}}\left(v_{2} v_{3}, v_{3} v_{4,}\right)=\mu\left(v_{2} v_{3}\right) \Lambda \mu\left(v_{3} v_{4}\right)=0.3 \Lambda 0.5=0.3
$$$$
\mu_{Q_{1} T_{f}}\left(v_{2} v_{3}, v_{4} v_{1}\right)=0
$$$$
\mu_{Q_{1} T_{f}}\left(v_{3} v_{4}, v_{4} v_{1}\right)=\mu\left(v_{3} v_{4}\right) \Lambda \mu\left(v_{4} v_{1}\right)=0.5 \Lambda 0.1=0.1
$$

Clearly, $\mu_{Q_{1} T_{f}}\left(v_{i}, v_{j}\right) \leq \sigma_{Q_{1} T_{f}}\left(v_{i}\right) \Lambda \sigma_{Q_{1} T_{f}}\left(v_{j}\right)$ for all $v_{i}, v_{j} \in V$ and hence the graph $Q_{1} T_{f}\left(\delta_{Q_{1} T_{f}}, \mu_{Q_{1} T_{f}}\right)$ is a fuzzy graph.

Th e graph $Q_{1} T_{f}=\left(\sigma_{Q_{1} T_{f}}, \mu_{Q_{1} T_{f}}\right)$ is a 1-quasi total fuzzy graph and its graph is as shown in figure 4.

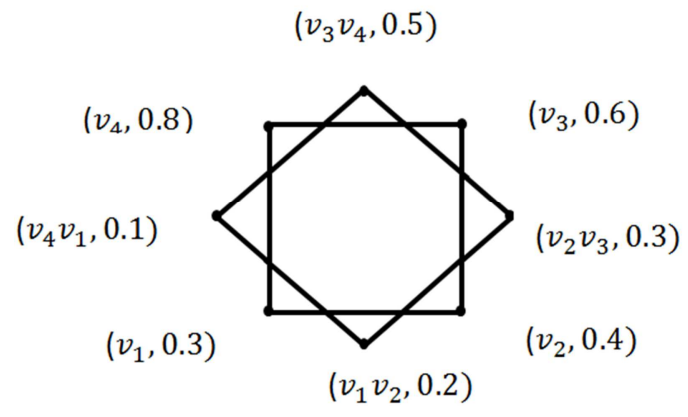

Figure 4. 1-Quasi fuzzy total graph.

\section{Properties of 1-Quasi Fuzzy Total Graph}

Theorem 4.1: Let $G=(V, \sigma, \mu)$ be a fuzzy graph.

$$
\operatorname{Order}\left(Q_{1_{T_{f}}}(G)\right)=\operatorname{Order}(G)+\operatorname{Size}(G) .
$$

Proof: By the definition of 1-quasi total fuzzy graph, the node set of $Q_{1} T_{f}(G)$ is $V U E$ and the fuzzy subset $\sigma_{Q_{1} T_{f}}(u)=$ $\sigma(u)$, if $u \in V$ and $\sigma_{Q_{1} T_{f}}(e)=\mu(e)$, if $e \in E$.

Now,

$$
\begin{gathered}
\operatorname{Order}\left(Q_{1} T_{f}(G)\right)=\sum_{u \in V \cup E} \sigma_{Q_{1} T_{f}}(u), \text { by definition }=\sum_{u \in V} \sigma_{Q_{1} T_{f}}(u)+\sum_{u \in E} \sigma_{Q_{1} T_{f}}(u)= \\
\sum_{u \in V} \sigma(u)+\sum_{u \in E} \sigma(u), \text { by definition of } Q_{1} T_{f}(u)=\operatorname{Order}(G)+\operatorname{Size}(G) .
\end{gathered}
$$

Note 1: For any fuzzy graph $G=(V, \sigma, \mu)$, $\operatorname{Order}(T(G))=\operatorname{Order}(G)+\operatorname{Size}(G)$. Where $T(G)$ is fuzzy total graph.

Theorem 4.2: Let $G=(V, \sigma, \mu)$ be a fuzzy graph, then

$$
\operatorname{Size}\left(Q_{1} T_{f}(G)\right)=\operatorname{Size}(G)+\sum_{e_{i,}, e_{j} \in E} \mu\left(e_{i}\right) \Lambda \mu\left(e_{j}\right)
$$

Proof: By definition of size of a fuzzy graph, we have;

$$
\begin{aligned}
& \qquad \operatorname{Size}\left(Q_{1} T_{f}\right)=\sum_{u, v \in V \cup E} \mu_{Q_{1} T_{f}}(\mathrm{u}, \mathrm{v})=\sum_{u, v \in V} \mu_{Q_{1} T_{f}}(\mathrm{u}, \mathrm{v})+\sum_{u \in V, e \in E} \mu_{Q_{1} T_{f}}(\mathrm{u}, \mathrm{e})+\sum_{\mathrm{e}_{\mathrm{i}}, \mathrm{e}_{\mathrm{j}} \in E} \mu_{Q_{1 T}}\left(\mathrm{e}_{\mathrm{i}}, \mathrm{e}_{\mathrm{j}}\right) \\
& =\sum_{u, v \in V} \mu_{Q_{1} T_{f}}(\mathrm{u}, \mathrm{v})+0+\sum_{\mathrm{e}_{\mathrm{i}}, \mathrm{e}_{\mathrm{j}} \in E} \mu_{Q_{1 T_{f}}}\left(\mathrm{e}_{\mathrm{i}}, \mathrm{e}_{\mathrm{j}}\right) \\
& \text { (The second summation is zero, since there is no fuzzy }=\operatorname{Size}(G)+\sum_{e_{i,}, e_{j} \in E} \mu\left(e_{i}\right) \Lambda \mu\left(e_{j}\right)
\end{aligned}
$$
relation between $u \in V$ and $e \in E$ in 1-quasi total fuzzy graph)

$$
=\sum_{u, v \in V} \mu(\mathrm{u}, \mathrm{v})+\sum_{\mathrm{e}_{\mathrm{i}}, \mathrm{e}_{\mathrm{j}} \in E} \mu\left(\mathrm{e}_{\mathrm{i}}, \mathrm{e}_{\mathrm{j}}\right)
$$

Note 2: For any fuzzy graph

$$
G=(V, \sigma, \mu), \operatorname{Size}(T(G))=
$$




$$
3 \operatorname{Size}(G)+\sum_{e_{i, e_{j} \in E}} \mu\left(e_{i}\right) \Lambda \mu\left(e_{j}\right) .
$$

Theorem 4.3: Let $G=(V, \sigma, \mu)$ be a fuzzy graph, then

$$
d\left(Q_{1} T_{f}(u)\right)=d_{G}(u), \text { if } u \in V
$$$$
d\left(Q_{1} T_{f(G)}(u)\right)=\sum_{u, v \in V} \mu_{Q_{1} T} f(G)(u, v)+\sum_{u \in V, e \in E} \mu_{Q_{1} T f(G)}(u, e),
$$

(where $\mathrm{u}$ lies on the edge of $e$ in the second summation).

$$
=\sum_{u, v \in V} \mu_{Q_{1} T_{f(G)}}(u, v)+0
$$

(The second summation is zero, since there is no fuzzy relation between $u \in V$ and $e \in E$ in 1-quasi total fuzzy graph)

$$
=\sum_{u, v \in V} \mu(u, v)=d_{G}(u) .
$$

Case 2: Let $e_{i} \in E$, then

$$
\begin{gathered}
d\left(Q_{1} T_{f(G)}\left(e_{i}\right)\right)=\sum_{u \in V} \mu_{Q_{1} T_{f(G)}}\left(e_{i}, v\right)+ \\
\sum_{e_{j} \in E} \mu_{Q_{1} T_{f(G)}}\left(e_{i}, e_{j}\right)=0+\sum_{e_{j} \in E} \mu_{Q_{1} T_{f(G)}}\left(e_{i}\right) \Lambda \mu_{Q_{1} T_{f(G)}}\left(e_{j}\right)
\end{gathered}
$$

(The first summation is zero, since there is no fuzzy relation between $u \in V$ and $e \in E$ in 1-quasi total fuzzy graph) $=$ bussy value of $e_{i}$ in $Q_{1} T_{f(G)}$.

Note 3: For any fuzzy graph, $G=(V, \sigma, \mu)$,

$$
\begin{gathered}
d\left(T_{G}(u)\right)=2 d_{G}(u), \text { if } u \in V \\
=\text { busy value of } e_{i} \text { in } T(G), \text { if } u \in E
\end{gathered}
$$

Theorem 4.4: 1-quasi total fuzzy graph of any fuzzy graph is disconnected graph.

Proof: Let, $G=(V, \sigma, \mu)$ be a fuzzy graph.

The fuzzy vertex set of $Q_{1} T_{f}(G)$ consists of $V \cup E$ of $G$ and the fuzzy vertex relation is defined only between $u, v \in V$ and $e_{i}, e_{j} \in E$. Since there is no fuzzy relation between $u \in V$ and $e \in E$ of elements in the vertex set of $Q_{1} T_{f}(G)$, then there is no path that connects $u$ and $e$ in $Q_{1} T_{f}(G)$ and $\mu^{\infty}(u, v)=0$.

Hence, $Q_{1} T_{f}(G)$ is disconnected graph.

\section{1-Quasi Fuzzy Total Coloring}

In this section we introduce the concept of 1-quasi fuzzy total coloring and discuss some of its properties.

Definition 5. (2): A family $\Gamma=\left\{\gamma_{1}, \gamma_{2}, \ldots, \gamma_{k}\right\}$, of a fuzzy set on $V \cup E$ is called a 1-quasi k-fuzzy total coloring of fuzzy graph, $G=(V, \sigma, \mu)$, if the following three conditions met.

i. $\operatorname{Max}\left\{\gamma_{i}(v)\right\}=\sigma(v)$ for all $v \in V$ and

$$
=\text { busy value of } e_{i} \text { in } Q_{1} T_{f}(G) \text {, if } u \in E
$$

Proof: By definition of degree of a vertex of a fuzzy graph given in definition 5 , we have the following two cases to prove the theorem.

Case 1: Let $u \in V$. Then,

$$
\operatorname{Max}\left\{\gamma_{i}(u, v)\right\}=\mu(u, v) \text { for all edges }(u, v) \in E .
$$

ii. $\gamma_{i} \Lambda \gamma_{j}=0$

iii.For every adjacent vertices $u, v$ of $Q_{1} T_{f}(G)$, $\operatorname{Min}\left\{\gamma_{i}(u), \gamma_{i}(v)\right\}=0$.

The least number of colors possible is called 1-quasi fuzzy total chromatic number of $Q_{1} T_{f}(G)$ and it is denoted by $\chi_{Q_{1}}^{f}(G)$.

Example 5.1: Consider a fuzzy graph $G=(V, \sigma, \mu)$ with vertex set $V=\left\{v_{1}, v_{2}, v_{3}, v_{4}, v_{5}, v_{6}\right\}$ and edge set $E=$ $\left\{v_{1} v_{2}, v_{2} v_{3}, v_{3} v_{4}, v_{4} v_{5}, v_{5} v_{6}, v_{6} v_{1}\right\}$, whose membership functions are defined as follows:

$$
\begin{gathered}
\sigma\left(v_{i}\right)=\left\{\begin{array}{l}
0.2, \text { for } i=1 \\
0.7, \text { for } i=2 \\
0.5, \text { for } i=3 \\
0.4, \text { for } i=4 \\
0.6, \text { for } i=5 \\
0.3, \text { for } i=6
\end{array}\right. \\
\mu\left(v_{i}, v_{j}\right)=\left\{\begin{array}{l}
0.2, \text { for }(i, j)=(1,2) \\
0.5, \text { for }(i, j)=(2,3) \\
0.1, \text { for }(i, j)=(3,4) \\
0.4, \text { for }(i, j)=(4,5) \\
0.3, \text { for }(i, j)=(5,6) \\
0.1, \text { for }(i, j)=(6,1)
\end{array}\right.
\end{gathered}
$$

Let us define a family of fuzzy sets $\Gamma=\left\{\gamma_{1}, \gamma_{2}\right\}$ on $V \cup E$ as follows:

$$
\begin{aligned}
& \gamma_{1}\left(v_{i}\right)=\left\{\begin{array}{l}
0.2, \text { for } i=1 \\
0.5, \text { for } i=3 \\
0.6, \text { for } i=5 \\
0, \text { Otherwise }
\end{array}\right. \\
& \gamma_{2}\left(v_{i}\right)=\left\{\begin{array}{l}
0.7, \text { for } i=2 \\
0.4, \text { for } i=4 \\
0.3, \text { for } i=6 \\
0, \text { Otherwise }
\end{array}\right.
\end{aligned}
$$

$$
\begin{gathered}
\gamma_{1}\left(v_{i}, v_{j}\right)=\left\{\begin{array}{c}
0.2, \text { for }(i, j)=(1,2) \\
0.1, \text { for }(i, j)=(3,4) \\
0.3, \text { for }(i, j)=(5,6) \\
0, \quad \text { Otherwise }
\end{array}\right. \\
\gamma_{2}\left(v_{i}, v_{j}\right)=\left\{\begin{array}{c}
0.5, \text { for }(i, j)=(2,3) \\
0.4, \text { for }(i, j)=(4,5) \\
0.1, \text { for }(i, j)=(6,1) \\
0, \quad \text { Otherwise }
\end{array}\right.
\end{gathered}
$$

Clearly, a family of fuzzy sets $\Gamma=\left\{\gamma_{1}, \gamma_{2}\right\}$ defined as 
above satisfies the definition of total coloring of fuzzy graphs and hence, $\chi_{T}^{f}(G)=2$.

When we come to our point of concern, we need to determine the chromatic number of 1-quasi total fuzzy graph of the fuzzy graph in the example 5.1.

Now, to construct a 1-quasi total fuzzy graph $Q_{1}(G)=$ $\left(V \cup E, \sigma_{Q_{1} T_{f}}, \mu_{Q_{1} T_{f}}\right)$, where

$V \cup E=\left\{v_{1}, v_{2}, v_{3}, v_{4}, v_{5}, v_{6}, v_{1} v_{2}, v_{2} v_{3}, v_{3} v_{4}, v_{4} v_{5}, v_{5} v_{6}, v_{6} v_{1}\right\}$

The fuzzy subset of $Q_{1} T_{f}(G)$ will be as follows;

$$
\begin{gathered}
\sigma_{Q_{1} T_{f}}\left(v_{i}\right)=\left\{\begin{array}{l}
0.2, \text { for } i=1 \\
0.7, \text { for } i=2 \\
0.5, \text { for } i=3 \\
0.4, \text { for } i=4 \\
0.6, \text { for } i=5 \\
0.3, \text { for } i=6
\end{array}\right. \\
\sigma_{Q_{1} T_{f}}\left(v_{i}, v_{j}\right)=\left\{\begin{array}{l}
0.2, \text { for }(i, j)=(1,2) \\
0.5, \text { for }(i, j)=(2,3) \\
0.1, \text { for }(i, j)=(3,4) \\
0.4, \text { for }(i, j)=(4,5) \\
0.3, \text { for }(i, j)=(5,6) \\
0.1, \text { for }(i, j)=(6,1)
\end{array}\right.
\end{gathered}
$$

The fuzzy relation will be:

$$
\begin{gathered}
\mu_{Q_{1} T_{f}}\left(v_{i}, v_{j}\right)=\left\{\begin{array}{l}
0.2, \text { for }(i, j)=(1,2) \\
0.5, \text { for }(i, j)=(2,3) \\
0.1, \text { for }(i, j)=(3,4) \\
0.4, \text { for }(i, j)=(4,5) \\
0.3, \text { for }(i, j)=(5,6) \\
0.1, \text { for }(i, j)=(6,1)
\end{array}\right. \\
\mu_{Q_{1} T_{f}}\left(v_{i} v_{j}, v_{j} v_{k}\right)=\left\{\begin{array}{l}
0.2, \text { for }(i j, j k)=(12,23) \\
0.1, \text { for }(i j, j k)=(23,34) \\
0.1, \text { for }(i j, j k)=(34,45) \\
0.3, \text { for }(i j, j k)=(45,56) \\
0.1, \text { for }(i j, j k)=(56,61) \\
0.1, \text { for }(i j, j k)=(61,12)
\end{array}\right.
\end{gathered}
$$

Let $\Gamma=\left\{\gamma_{1}, \gamma_{2}\right\}$ be a family of fuzzy subset defined on $V \cup E$ as follows:

For the vertex set;

$$
\gamma_{1}\left(v_{i}\right)=\left\{\begin{array}{l}
0.2, \text { for } i=1 \\
0.5, \text { for } i=3 \\
0.6, \text { for } i=5 \\
0, \text { Otherwise }
\end{array}\right.
$$

$$
\begin{gathered}
\gamma_{1}\left(v_{i} v_{j}\right)=\left\{\begin{array}{l}
0.2, \text { for } i j=12 \\
0.1, \text { for } i j=34 \\
0.3, \text { for } i j=56 \\
0, \text { Otherwise }
\end{array}\right. \\
\gamma_{2}\left(v_{i}\right)=\left\{\begin{array}{l}
0.7, \text { for } i=2 \\
0.4, \text { for } i=4 \\
0.3, \text { for } i=6 \\
0, \text { Otherwise }
\end{array}\right. \\
\gamma_{2}\left(v_{i} v_{j}\right)=\left\{\begin{array}{c}
0.5, \text { for }(i, j)=(2,3) \\
0.4, \text { for }(i, j)=(4,5) \\
0.1, \text { for }(i, j)=(6,1) \\
0, \quad \text { Otherwise }
\end{array}\right.
\end{gathered}
$$

For the edge set;

$$
\begin{gathered}
\gamma_{1}\left(v_{i}, v_{j}\right)=\left\{\begin{array}{l}
0.2, \text { for }(i, j)=(1,2) \\
0.1, \text { for }(i, j)=(3,4) \\
0.3, \text { for }(i, j)=(5,6) \\
0, \quad \text { Otherwise }
\end{array}\right. \\
\gamma_{1}\left(v_{i} v_{j}, v_{j} v_{k}\right)=\left\{\begin{array}{l}
0.2, \text { for }(i j, j k)=(12,23) \\
0.1, \text { for }(i j, j k)=(34,45) \\
0.1, \text { for }(i j, j k)=(56,61)
\end{array}\right. \\
\gamma_{2}\left(v_{i} v_{j}\right)=\left\{\begin{array}{c}
0, \text { Otherwise } \\
0.4, \text { for }(i, j)=(2,3) \\
0.1, \text { for }(i, j)=(4,5)=(6,1) \\
0, \quad \text { Otherwise }
\end{array}\right. \\
\gamma_{2}\left(v_{i} v_{j}, v_{j} v_{k}\right)=\left\{\begin{array}{c}
0.1, \text { for }(i j, j k)=(23,34) \\
0.3, \text { for }(i j, j k)=(45,56) \\
0.1, \text { for }(i j, j k)=(61,12)
\end{array}\right. \\
\quad 0, \quad \text { Otherwise }
\end{gathered}
$$

Using table 1 below we can check whether $\Gamma$ satisfies the definition of 1-quasi total coloring of $G$.

Table 1. Example of 1-quasi total coloring of a fuzzy graph $G=(V, \sigma, \mu)$.

\begin{tabular}{llllll}
\hline $\boldsymbol{v}$ and $\boldsymbol{e}$ & $\boldsymbol{\gamma}_{\mathbf{1}}$ & $\boldsymbol{\gamma}_{\mathbf{2}}$ & Max. & $\boldsymbol{\gamma}_{\mathbf{1}} \boldsymbol{\Lambda} \boldsymbol{\gamma}_{\mathbf{2}}$ \\
\hline$v_{1}$ & 0.2 & 0 & 0.2 & 0 & Min. \\
$v_{2}$ & 0 & 0.7 & 0.7 & 0 & $\operatorname{Min}\left\{\gamma_{i}\left(v_{1}\right), \gamma_{i}\left(v_{2}\right)\right\}=0$ \\
$v_{3}$ & 0.5 & 0 & 0.5 & 0 & $\operatorname{Min}\left\{\gamma_{i}\left(v_{2}\right), \gamma_{i}\left(v_{3}\right)\right\}=0$ \\
$v_{4}$ & 0 & 0.4 & 0.4 & 0 & $\operatorname{Min}\left\{\gamma_{i}\left(v_{3}\right), \gamma_{i}\left(v_{4}\right)\right\}=0$ \\
$v_{5}$ & 0.6 & 0 & 0.6 & 0 & $\operatorname{Min}\left\{\gamma_{i}\left(v_{4}\right), \gamma_{i}\left(v_{5}\right)\right\}=0$ \\
$v_{6}$ & 0 & 0.3 & 0.3 & 0 & $\operatorname{Min}\left\{\gamma_{i}\left(v_{5}\right), \gamma_{i}\left(v_{6}\right)\right\}=0$ \\
$v_{1} v_{2}$ & 0.2 & 0 & 0.2 & 0 & $\operatorname{Min}\left\{\gamma_{i}\left(v_{6}\right), \gamma_{i}\left(v_{1}\right)\right\}=0$ \\
$v_{2} v_{3}$ & 0 & 0.5 & 0.5 & 0 & $\operatorname{Min}\left\{\gamma_{i}\left(v_{1} v_{2}\right), \gamma_{i}\left(v_{2} v_{3}\right)\right\}=0$ \\
$\left.\left.v_{3} v_{4} v_{3}\right), \gamma_{i}\left(v_{3} v_{4}\right)\right\}=0$ \\
$v_{4} v_{5}$ & 0.1 & 0 & 0.1 & 0 & $\operatorname{Min}\left\{\gamma_{i}\left(v_{3} v_{4}\right), \gamma_{i}\left(v_{4} v_{5}\right)\right\}=0$ \\
\hline
\end{tabular}




\begin{tabular}{|c|c|c|c|c|c|}
\hline$v$ and $e$ & $\gamma_{1}$ & $\gamma_{2}$ & Max. & $\gamma_{1} \Lambda \gamma_{2}$ & Min. \\
\hline$v_{5} v_{6}$ & 0.3 & 0 & 0.3 & 0 & $\operatorname{Min}\left\{\gamma_{i}\left(v_{5} v_{6}\right), \gamma_{i}\left(v_{6} v_{1}\right)\right\}=0$ \\
\hline$v_{6} v_{1}$ & 0 & 0.1 & 0.1 & 0 & $\operatorname{Min}\left\{\gamma_{i}\left(v_{6} v_{1}\right), \gamma_{i}\left(v_{1} v_{2}\right)\right\}=0$ \\
\hline$\left(v_{1}, v_{2}\right)$ & 0.2 & 0 & 0.2 & 0 & \\
\hline$\left(v_{2}, v_{3}\right)$ & 0 & 0.5 & 0.5 & 0 & \\
\hline$\left(v_{3}, v_{4}\right)$ & 0.1 & 0 & 0.1 & 0 & \\
\hline$\left(v_{4}, v_{5}\right)$ & 0 & 0.4 & 0.4 & 0 & \\
\hline$\left(v_{5}, v_{6}\right)$ & 0.3 & 0 & 0.3 & 0 & \\
\hline$\left(v_{6}, v_{1}\right)$ & 0 & 0.1 & 0.1 & 0 & \\
\hline$\left(v_{1} v_{2}, v_{2} v_{3}\right)$ & 0.2 & 0 & 0.2 & 0 & \\
\hline$\left(v_{2} v_{3}, v_{3} v_{4}\right)$ & 0 & 0.1 & 0.1 & 0 & \\
\hline$\left(v_{3} v_{4}, v_{4} v_{5}\right)$ & 0.1 & 0 & 0.1 & 0 & \\
\hline$\left(v_{4} v_{5}, v_{5} v_{6}\right)$ & 0 & 0.3 & 0.3 & 0 & \\
\hline$\left(v_{5} v_{6}, v_{6} v_{1}\right)$ & 0.1 & 0 & 0.1 & 0 & \\
\hline$\left(v_{6} v_{1}, v_{1} v_{2}\right)$ & 0 & 0.1 & 0.1 & 0 & \\
\hline
\end{tabular}

As shown in the table $1 \Gamma=\left\{\gamma_{1}, \gamma_{2}\right\}$ satisfies the definition of 1-quasi fuzzy total coloring of fuzzy graph $G$.

Therefore, $\chi_{Q_{1} T}^{f}(G)=2$.

\section{Conclusion}

In this article we have defined 1-quasi total fuzzy graph for a given fuzzy graph. For this concept to be clear we constructed a fuzzy graph and from this graph 1-quasi total fuzzy graph is developed and its graph is sketched. For easy understanding the graph of a given fuzzy graph and its 1quasi total fuzzy graph is given. For the sake of making the study to be complete, theorems regarding properties of total fuzzy graph are established for 1-quasi total fuzzy graph and the results obtained from the proof of the theorem are compared against the results for total fuzzy graphs. Moreover, we have defined 1-quasi total coloring for fuzzy graph and its total coloring is exemplified.

\section{References}

[1] Total Equitable Domination in Fuzzy Graphs. Rani, K. M. Dharmalingam and M. 2016, Bulletin of the International Mathematical Virtual Institute, Vol. 6, pp. 49-54.

[2] Fuzzy Total Coloring and Chromatic Number of Complete Fuzzy Graph. V. Nevethana, A. Parvathi. 3, 2013, International Journal of Engineering and Development, Vol. 6, pp. 377-384.

[3] R. Balakrishina, K. Ranganathan. A Text Book of Graph Theory. New York: Springer-Verlag, 2000.

[4] J. A. Bondy, U. S. R. Murthy. Graph Theory with Applications. s. 1.: The Macmillan Press Ltd, 1976.

[5] Graphs and their Chromatic Numbers. Behzad, M. s. 1.: Michigan State University, 1965, Doctoral Thesis.

[6] Harary, F. Graph Theory. s. 1.: Addison-Wesley Publishing Company, 1972.
[7] Graph Equations for Line Graphs, Total Graphs, Middle Graphs and Quasi-Total Graphs. D. V. S. Sastry, B. Syam Prasad Raju. 1984, Discrete Mathematics, Vol. 48, pp. 113119.

[8] A Discussion for Bounds for 1-quasi Total Colourings. R. V. N. Sirnvasarao, J. VenkateswaraRao. 6, 2012, International Journal of Mathematical Archive, Vol. 3, pp. 2314-2320.

[9] Fuzzy Graphs, In Fuzzy Sets and their Application to Cognitive and Decision Process. Rosenfield, A. New York: Academic Press, 1975, pp. 77-95.

[10] Fuzzy Sets, Information and Control. A. Zadeh, Lofti. 1965, Vol. 8, pp. 338-353.

[11] Vertex Strength of Fuzzy Graphs. Eslahchi, B. N. Onagh. 2006, International Journal of Mathematics and Mathematical Science.

[12] Fuzzy Total Coloring of Fuzzy Graphs. S. Lavanya, R. Sattanathan. 3, 2009, International Journal of Information Technology and Knowledge Management, Vol. 2, pp. 37-39.

[13] Fuzzy Cgromatic Number of Line, Total and Middle Graphs of Fuzzy Complete Graphs. S. Kavitha, S. Lavanya. 2, 2014, Annas of Pure and Applied Mathematics, Vol. 8, pp. 251-260.

[14] J. N. Morderson, P. S. Nair. Fuzzu Graphs and Fuzzy Hypergraphs. s. 1.: Physica-Verlag, 2000.

[15] Operations on fuzzy graphs. Peng, J. N. Mordeson and C. S. S. 1.: Information Sciences, 1994, Vol. 79, pp. 159-170.

[16] Some Remarks on Fuzzy Graphs. Bhattacharya, P. 5, s. 1.: Pattern Recognition Letter, 1987, Vol. 6, pp. 297-302.

[17] Chromatic Number of Resultant of Fuzzy Graphs. Sunitha, Anjaly Kishore and M. S. s. 1.: Elsevier, 2016, Fuzzy Information and Engineering, Vol. 8.

[18] Total Chromatic Number of Middle and Total Graph of Path and Sunlet Graph. Jayaraman, D. Muthuramakrishnan G. 4, 2018, International Journal of Scientific and Innovative Mathematical Research (IJSIMR), Vol. 6, pp. 1-9. 